\title{
UCRL-PROC-216929
}

LAW RENCE LIVERMORE N A T IO N A L LABORATORY

\section{A Summary of Recent Damage-Initiation Experiments on KDP Crystals}

C. W. Carr, M. D. Feit, A. M. Rubenchik, J. B. Trenholme, M. L. Spaeth

November 8, 2005

Boulder Damage Symposium XXXVII Boulder, CO, United States September 19, 2005 through September 22, 2005 
This document was prepared as an account of work sponsored by an agency of the United States Government. Neither the United States Government nor the University of California nor any of their employees, makes any warranty, express or implied, or assumes any legal liability or responsibility for the accuracy, completeness, or usefulness of any information, apparatus, product, or process disclosed, or represents that its use would not infringe privately owned rights. Reference herein to any specific commercial product, process, or service by trade name, trademark, manufacturer, or otherwise, does not necessarily constitute or imply its endorsement, recommendation, or favoring by the United States Government or the University of California. The views and opinions of authors expressed herein do not necessarily state or reflect those of the United States Government or the University of California, and shall not be used for advertising or product endorsement purposes. 


\title{
A Summary of Recent Damage-Initiation Experiments on KDP Crystals
}

\author{
C. W. Carr , M. D. Feit, A. M. Rubenchik, \\ J. B. Trenholme, M. L. Spaeth \\ Lawrence Livermore National Laboratory \\ 7000 East Avenue, Mailstop L-592 \\ Livermore, CA 94550
}

\begin{abstract}
We summarize recent investigations of the density and morphology of bulk damage in KDP crystals as a function of pulse duration, temporal profile, wavelength, and energy fluence. As previously reported by Runkel et al. ${ }^{1}$, we also find that the size of bulk damage sites varies roughly linearly with pulse duration for pulses between $1 \mathrm{~ns}$ and $9 \mathrm{~ns}$. However this trend no longer applies at pulse durations below $1 \mathrm{~ns}$. Experiments measuring the damage density and size distribution as a function of wavelength confirm many previous works which indicated a strong dependence of damage density with wavelength. However, we also find that the size of damage sites is relatively insensitive to wavelength. Further we see damage due to Flat-In-Time (FIT) pulses has different pulse length and fluence dependence than Gaussian pulses. We demonstrate that a simple thermal diffusion model can account for observed differences in damage densities due to square and Gaussian temporally shaped pulses of equal fluence. Moreover, we show that the key laser parameter governing size of the bulk damage sites is the length of time the pulse remains above a specific intensity. The different dependences of damage density and damage site size on laser parameters suggest different absorption mechanisms early and late in the damaging pulse.
\end{abstract}

\section{INTRODUCTION}

KDP and DKDP crystals are used in frequency converters and Pockel cells in both table-top and large-aperture laser systems. Laser-induced bulk damage in these materials scatters light and increases beam contrast, which in turn can increase the likelihood of damage to down stream optics. Avoiding laser-induced bulk damage constrains system design and limits system performance. ${ }^{2}$ It is therefore of significant interest to understand this phenomenon in order to prevent, reduce, and/or manage bulk damage in KDP and DKDP crystals. Bulk damage in KDP and DKDP crystals consists of small micro cavities, commonly referred to as "pinpoint" bulk damage sites. These damage sites tend not to grow with repeated laser irradiation ${ }^{3}$, but there can be many such sites. A limiting factor for laser operation is scattering from the bulk damage sites, which can cause significant down stream modulation on a propagating laser beam for pinpoint densities as low as a few tens per $\mathrm{mm}^{3}$. The density at which bulk damage becomes detrimental to laser performance depends on the size of the pinpoints and their optical cross-section. Thus, it is important to establish how the size and density of bulk pinpoints are affected by laser parameters such as pulse shape, pulse duration, wavelength, and fluence.

Although no work has been previously dedicated to studying the morphology of these damage sites, several observations in the literature depict them as voids, occasionally surrounded by cracks along the crystalline axis. ${ }^{4-6}$ Our observations reported here indicate that DKDP bulk damage sites have a complicated morphology, which depends on the laser parameters by which the bulk damage was created. Figure 1 depicts measurements of bulk damage sites by bright field microscopy, SEM, and 2D Micro-Raman spectroscopy. From the three measurements we see that bulk damage sites consist of a semi-void core containing recrystallized or rubbleized

*Correspondence: carr19@1lnl.gov, (925) 422-8755 
material surrounded by a shell of modified material, which may be crystalline in nature. We hypothesize that this modified material is somewhat densified due to passage of the shockwave accompanying the damage initiation. ${ }^{5,7}$ The micro-Raman spectroscopy confirms that the shell surrounding the core of the damage site is altered from undamaged DKDP.

In the past, damage site size has been measured with scattering of coherent light. For example, in 2001 Runkel et al. ${ }^{1}$ showed that damage sites in DKDP increased roughly linearly in size for pulse durations from 1 ns to $9 \mathrm{~ns}$ (see figure 1). When we repeated these measurements using white light scattering as our illumination source we observed the same general trend but found the pinpoints to be substantially smaller than reported previously (see figure 1). We have shown previously that the discrepancy in these two observations can be attributed to the illumination sources used ${ }^{8}$. Coherent light sources will make some, but not all, pinpoints appear larger. This is likely due to the enhanced coupling between the coherent light and the stressed material surrounding the pinpoint core (see figure 2). In the present work the size of pinpoints will be considered to be that of the core as observed in bright field microscopy.
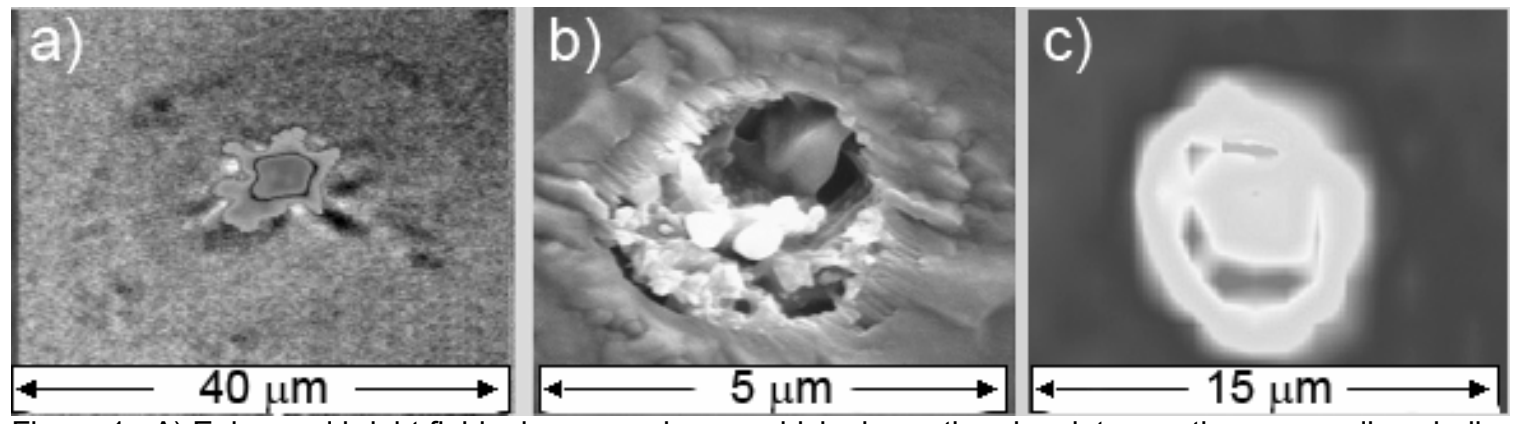

Figure 1. A) Enhanced bright field microscope image which shows the pinpoint core, the surrounding shell and a subtly modified region. B) SEM image of a pinpoint showing the core and shell. C) 2D micro-Raman map of the $716 \mathrm{~cm}^{-1}$.

The localized nature of the pinpoints indicates the presence of an extrinsic precursor. In a study of laser-induced damage probability as a function of wavelength, we found that the fluence needed for constant damage probability had two sharp steps at photon energies close to fractions of the band gap in DKDP ${ }^{9}$. The localized nature of the damage is one of the observations that suggest that the initial energy absorption mechanism is defect-assisted multi-photon / multi-step absorption ${ }^{9}$. We have previously demonstrated that during the damage process, peak transient pressures and temperatures on the order of 300 kbar $10,000 \mathrm{~K}$, respectively, are reached. ${ }^{7}$ Several models describing the initial energy absorption have been put forward though none fully accounts for these and other aspects of laser-induced damage ${ }^{9-11}$, indeed in this work we will argue that the differences of how pinpoint density and pinpoint size depend on laser parameters indicate that multiple energy absorption mechanisms are dominant at various times during the laser-induced damage process.

\section{EXPERIMENTAL TECHNIQUE}

To measure the damage density as a function of fluence $(\rho(\phi))$ composed at a single wavelength; a pulse from a large-aperture $(\sim 1-\mathrm{cm}$ diameter) beam is used to induce damage in a sample crystal. A high-resolution image of the fluence near-field is recorded. A dark-field visible light scatter map (DMS) of the damage produced by the laser pulse is then collected with a digital camera. By registering fluence and calibrated damage images we can correlate local fluence to corresponding local damage levels thus determining $\rho(\phi)$. The method consists of four basic steps; a) damage initiation (sample preparation), b) dark-field scatter measurement, c) registration of the fluence near field to the dark field scatter measurement, and d) calibration of scatter intensity in terms of pinpoint size and density. 


\section{A. Damage initiation}

A sample is exposed to a pulse from a slowly focusing beam $(f / 10)$. A wedge in the beam line before the sample but after the focusing lens is used to produce two $\sim 4 \%$ reflections. The reflections off the front and rear surface of the wedge are incident on a CCD camera (in a plane equivalent to the rear surface of the sample) and pyrolectric calorimeter, respectively. In conjunction the two measurements are used to produce a calibrated fluence profile of the beam incident on the sample for use in the damage density image analysis. The beam continues on to the sample producing damage primarily in the bulk. The local spatial variations of laser beam fluence will produce corresponding varying bulk damage densities in the sample. This spatial correlation of fluence and damage density is possible because the correlation length of fluence fluctuations is a few hundred microns while the fundamental unit of damage (the pinpoint) is on the order of $5-10 \mu \mathrm{m}$ in diameter.

\section{B. Dark-field scatter measurement}

Dark-field imaging can easily be used to measure scatter from damage density. The Damage Mapping System (DMS) ${ }^{12}$ measures the large angle scatter produced by the pinpoints. In brief, this is accomplished by illuminating the sample through the edges perpendicular to a camera and recording a dark-field image (see figure 1) with a digital camera with approximately $35-\mu \mathrm{m}$ resolution. Although individual pinpoints cannot be resolved at this resolution, changes in damage density on the scale of $35 \mu \mathrm{m}$ or larger can be.

\section{Registration of scatter image and fluence distribution}

Registering the fluence at the sample (the near-field image) to the DMS dark field scatter image is greatly simplified by careful determination of the magnification and orientation of both the fluence near-field and DMS dark field cameras. With known magnifications and relative orientations, registration of fluence and damage images is easily accomplished in any image manipulation program in which partly opaque layers may be overlaid and independently adjusted in both scale and by relative rotation. The pixels of the fluence image can be calibrated to absolute fluence units (within uncertainty of $7 \%$ ) of $\mathrm{J} / \mathrm{cm}^{2}$ by first dividing the pulse energy by net counts (observed counts minus background counts) recorded by all pixels on the CCD to determine the energy per count. The fluence at a given pixel is then calculated by multiplying the energy per count by the number of counts in the pixel and dividing by the area of the pixel. Once the images are registered, forming ordered pairs from the values in corresponding pixels of each image generates a 'scatter' vs. 'fluence' curve.

\section{Calibration of scatter to pinpoint density}

When quantitative information is required, it is necessary to count the pinpoints and relate their density to fluence. In a large $(0.5 \mathrm{~m} \times 0.5 \mathrm{~m})$ optic as many as $10^{8}$ pinpoints may be tolerated before scattered light significantly affects the beam contrast, making individual measurements of each pinpoint a practical impossibility. With pinpoints on the order of $5 \mu \mathrm{m}$ in diameter, the relationship between scatter and pinpoints density of a region should be linear, in which case a statistical sampling of pinpoint densities can be taken to calibrate luminosity (scatter) to pinpoint density in the DMS map as demonstrated below. In principle this can be accomplished by counting the pinpoints in a single small region with an optical microscope with the only difficulty lying in accurate registration with the DMS image. This may be accomplished by careful measurements of the distance from multiple features predominate in the DMS image and easily found with the microscope (such as edges of the part or surface damage sites).

Damage site density and morphology was also studied in a converting crystal where the first three harmonics of the laser where simultaneously present. The effect of multiple simultaneous wavelengths on damage density was studied with Gaussian pulses only. In this set of 
experiments a DKDP tripler cut crystal was damaged while actively converting. The input and output fluences were measured and the local $1 \omega, 2 \omega$, and $3 \omega$ fluences were calculated as a function of depth within the crystal. The modeling ${ }^{13}$ of the frequency conversion includes several physical processes: 3-wave mixing, bulk loss, 2 photon absorption, diffraction, non-linear susceptibility bulk loss, and walk-off. . Optical properties of the converter crystal are specified by its composition, and geometry. The damage site size and density are then measured with an automated microscope in a small $\left(\sim 2 \times 2 \mathrm{~mm}^{2}\right)$ region where the total fluence varied by only $\sim 0.5$ $\mathrm{J} / \mathrm{cm}^{2}$.

\section{RESULTS}

Pinpoint density is studied as a function of fluence for Flat-In-Time (FIT) and Gaussian pulses. In order to ameliorate any effect by crystal variability, adjacent sites on a single DKDP crystal were damage tested with a Gaussian and a FIT pulse, respectively. The damage density as a function of fluence for each pulse shape is plotted in figure 2 . The onset of detectable damage is seen to be at $2.5 \mathrm{~J} / \mathrm{cm}^{2}$ for a FIT pulse and $3.5 \mathrm{~J} / \mathrm{cm}^{2}$ for a Gaussian pulse. The temporal shape of each pulse may be seen in the inset of figure 2. In addition at any given fluence above 3.5 $\mathrm{J} / \mathrm{cm}^{2}$ the damage produced by the FIT pulse is observed to be significantly denser than the damage produced by the Gaussian pulse at the same fluence.

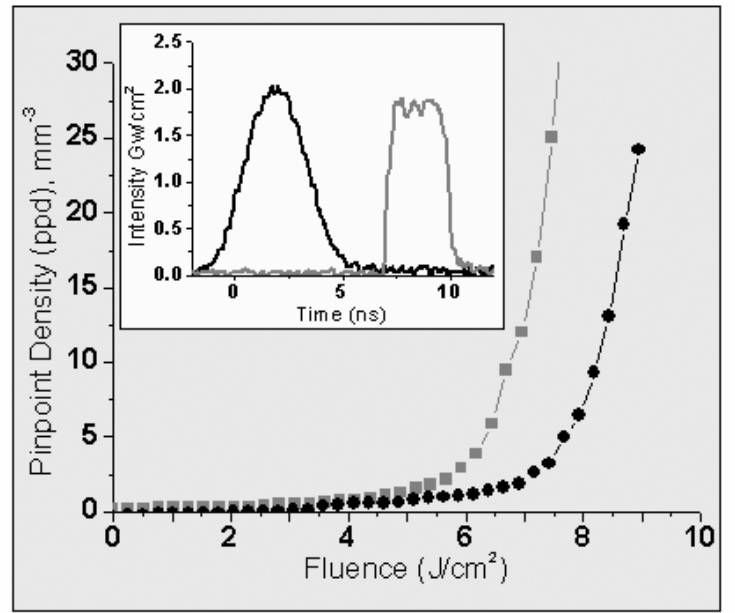

Figure 2. Measured damage densities as a function of fluence $(\rho(\phi))$ for FIT and Gaussian pulses. The inset depicts the temporal pulse shape of both pulses

The calculated average $1 \omega, 2 \omega$, and $3 \omega$ fluences can be seen as a function of depth in figure 3 . A $9 \mathrm{~mm}$ thick Type II DKDP Tripler cut converter was used for these measurements. Calculations by J.M. Auerbach predicted that the local $3 \omega$ fluence reached a maximum not at the output face of the crystal but at a depth of approximately $7 \mathrm{~mm}$ from the input face. At a depth of $7 \mathrm{~mm}$, the model predicted the start of back conversion and the $3 \omega$ fluence was less than its peak value at the exit of the crystal. 


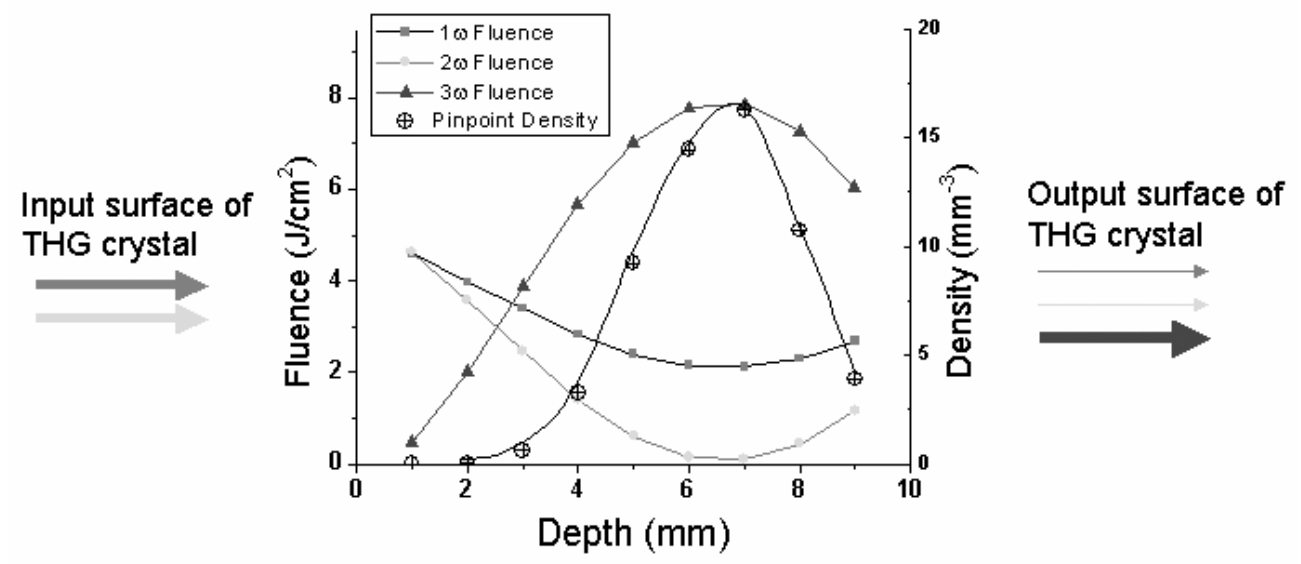

Figure 3. Calculated fluence as a function of depth in a converting crystal and measured pinpoint density as a function of depth.

The pinpoint density and size were also measured as a function of depth in the converting tripler. The size of the damage sites was observed to be nearly constant throughout the depth of the crystal. The density however is seen in figure 3 to vary substantially as a function of depth. The peak of the damage density is observed to be at a depth of $\sim 7 \mathrm{~mm}$ in from the entrance face of the crystal. This is in rough correspondence to the calculated location of the peak $3 \omega$ fluence.

Because the damage density and the fluence of each wavelength have been determined at the same depths, the damage density can be plotted as a function of local $1 \omega, 2 \omega$, and $3 \omega$ fluences (see figure 4). The pinpoint density as a function of fluence as measured in a separate experiment using a GAUSSIAN $3 \omega$ pulse of the same pulse duration and is also shown in figure 4.

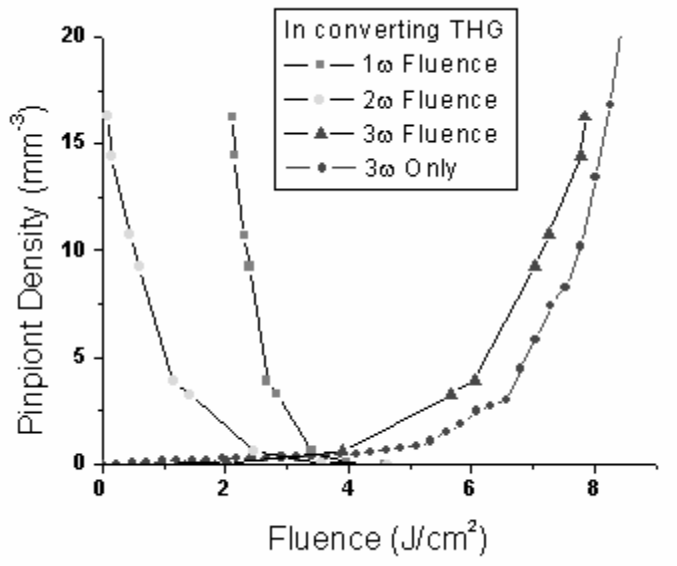

Figure 4. Damage densities as a function of local $1 \omega, 2 \omega$, and $3 \omega$ fluence is plotted by matching each calculated local fluence at each depth to the damage density measured at that depth. The damage density as a function of $3 \omega$ fluence without the other light present is also plotted.

The plots of pinpoint densities as a function of both $1 \omega$ and $2 \omega$ fluence appear to indicate that the damage density is reduced with increasing fluence but that the damage density increases with local $3 \omega$ fluence. It must be emphasized that the damage density is being plotted as a function of only one component of the local fluence simultaneously present in the crystal. Thus the reciprocal relationship between damage density and $1 \omega$ and $2 \omega$ fluences merely indicate that these wavelengths are not the predominate cause of damage initiation in a converting tripler. 
This interpretation is supported by the strong correlation between increasing $3 \omega$ fluence and damage density in the converting crystal.

Even though the damage in the converting tripler is clearly dominated by the presence of $3 \omega$ light, comparison to the damage density produced in the $3 \omega$ only experiment indicate that the damage density is somewhat higher in the presence of the $1 \omega$ and $2 \omega$ light. This indicates that although the $3 \omega$ light dominates damage initiation, there is some additive effect produced by the $1 \omega$ and/or $2 \omega$ light. It is also interesting to note that there is no damage produced by the $1 \omega$ and $2 \omega$ light when they are at their largest values but no $3 \omega$ light is present. This implies that the increase in damage density observed in the converting experiment is an interaction between the multiple wavelengths and not a linear addition of damage caused by each wavelength alone. Furthermore at the higher $3 \omega$ fluences the damage density in the converting crystal approaches that seen in the $3 \omega$ only experiment. Because there is still significant amounts of $1 \omega$ light present, but very little $2 \omega$ light it is likely that the enhancement to damage density is due largely if not entirely to the presence of the $2 \omega$ light.

This strong wavelength dependence on damage site density is in contrast to that observed for pinpoint size. The observation that the pinpoint size was relatively constant though the depth of the crystal indicates that the total fluence governs the size of the pinpoints. In Figure 5 the pinpoint size in the converting crystals is plotted vs. the total fluence (the sum of $1 \omega, 2 \omega$, and $3 \omega$ ). Also the pinpoint size from the $3 \omega$ only experiment is plotted vs. the total fluence in that experiment ( $3 \omega$ light only).

The measurements made here concerning pinpoint densities in the presence of single-color fluences are consistent with the picture ${ }^{14}$ of an initial distribution of tiny absorbing precursors. This idea has been further developed in two other papers ${ }^{15,16}$ in these proceedings. This model has been reasonably successful in explaining trends seen in damage initiation quantities such as threshold fluence scaling, dependence on pulse shape, etc. We have shown that damage density depends on pulse shape as well as pulselength. We hypothesize that this is true because different pulse shapes produce different thermal histories. For example, heating with a FIT pulse leads to a continual increase in temperature over the entire pulse duration, whereas heating with a Gaussian pulse results in maximum temperature near the center of the pulse. Energy contained in the Gaussian pulse past this time does not contribute to setting the maximum temperature and therefore producing damage. The mathematical ideas of pulse shape dependence of thermal effects have been embodied in a code called Equivinit (by J.B. Trenholme) that calculates the accumulated heat present in the damage precursor as a function of pulse shape and duration. By assuming that a precursor need only reach some peak temperature for damage to become unavoidable, Equivinit can be used to estimate the damage density as a function of fluence from an arbitrarily shaped pulse from the measured $\rho(\phi)$ of a Gaussian pulse and a single adjustable parameter which is equivalent to the pulse scaling exponent. 
In figure 6 a prediction $\rho(\phi)$ for a FIT pulse based on the measured $\rho(\phi)$ for a Gaussian pulse and a pulse scaling parameter of $\tau^{0.35}$ is shown. This is consistent with the $3 \omega$ pulse scaling parameter reported previously by Runkel ${ }^{1}$.

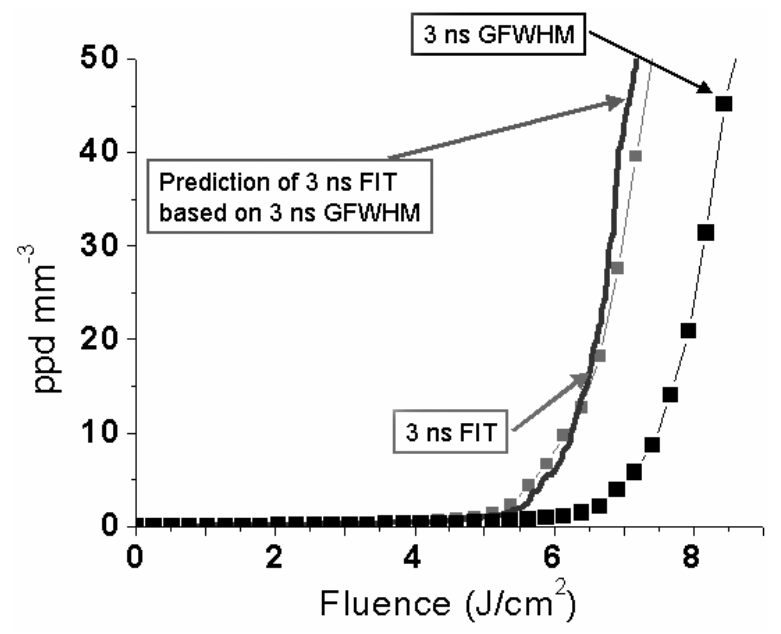

Figure 6. Damage density as a function of fluence for FIT and GAUSSIAN pulses. The $\rho(\phi)$ for a FIT pulse is predicted from the $\rho(\phi)$ measured for a GAUSSIAN pulse of the same full width half-maximum pulse duration.

The new observations reported here concerning dependence of pinpoint size and density on wavelength, fluence and pulselength offer constraints on any theoretical description that goes beyond initiation since ultimate size depends on how much energy is coupled into the optical material. Once a dense solid-state plasma is formed during initiation, it is capable of absorbing different wavelengths efficiently. The fact that pinpoint size for Gaussian pulses scales essentially linearly with energy fluence might suggest there is a threshold intensity for sustained pinpoint growth once initiation occurs.

\section{SUMMARY}

In conclusion, we made a series of measurements to determine the effect of pulse shape, duration and wavelength on bulk damage density and size in KDP and DKDP crystals. We have observed that the density of pinpoints is strongly dependant on fluence, pulse shape, duration and wavelength. The high sensitivity of damage density to wavelength is consistent with damage probabilities measured for a wide range of single wavelengths ${ }^{9}$.

In contrast, the size of pinpoints are insensitive to wavelength, which suggests that two different energy deposition mechanisms govern the initiation of damage and the growth of the pinpoint. If this thinking is generalized to the entire laser-induced damage process it may explain why several simple models (each of which assume only one absorption mechanism) explain aspects of damage behavior well, but fail for others ${ }^{9,17,18}$.

For example the defect assisted multi-photon or multi-step absorption model is well suited to explain the wavelength dependence of damage density but can account for the effect of pulse duration only with difficulty. On the other hand, the small absorber model explains the effect of pulse duration and pulse shape by assuming that precursors of different sizes will be most susceptible to damage under different conditions such as pulselength. It would be desirable to combine aspects of these two models to describe a wider range of experimental observations. Just how to do this is not yet clear, however the general approach appears to be assuming that the initial energy deposition occurs by defect assisted multi-photon absorption in precursors of different size and defect density. The initial energy absorbed at each precursor may then 
produces regions of moderate density solid-state plasma of varying sizes. These regions would intern act as weakly absorbing particles, similar to those described else ware ${ }^{15,16}$.

\section{ACKNOWLEDGEMENTS}

This work was performed under the auspice of the U.S. Department of Energy by the University of California, Lawrence Livermore National Laboratory under contract No. W-7405-Eng-48

\section{REFERENCES}

1 M. Runkel, A. Burnham, D. Milam, W. Sell, M. D. Feit, and A. M. Rubenchik, SPIE 4347, 359 (2001).

C. W. Carr, M. D. Feit, M. Nostrand, and J. J. Adams, Phys. Rev. B Submitted (2005).

C. W. Carr, M. Staggs, H. B. Radousky, and S. G. Demos, SPIE 4932, 429 (2003).

A. K. Burnham, M. Runkel, M. D. Feit, A. M. Rubenchik, R. L. Floyd, T. A. Land, I

W. J. Siekhaus, and R. A. Hawley-Fedder, Applied Optics 42, 5483 (2003).

H. Jiang, J. McNary, H. W. K. Tom, M. Yan, H. B. Radousky, and S. G. Demos, Applied Physics Letters 81, 3149 (2002).

H. Yoshida, T. Jitsuno, H. Fujita, M. Nakatsuka, M. Yoshimura, T. Sasaki, and K. Yoshida, Applied Physics B-Lasers and Optics 70, 195 (2000).

C. W. Carr, H. B. Radousky, A. M. Rubenchik, M. D. Feit, and S. G. Demos, Physical Review Letters 92, 087401 (2004).

C. W. Carr, M. D. Feit, and A. Rubenchik, SPIE 5647, 532 (2004).

C. W. Carr, H. B. Radousky, and S. G. Demos, Physical Review Letters 91, 127402 (2003).

B. C. Stuart, M. D. Feit, A. M. Rubenchik, B. W. Shore, and M. D. Perry, Physical Review Letters 74, 2248 (1995).

M. D. Feit, A. M. Rubenchik, and M. Runkel, SPIE 4347, 383 (2001).

F. Rainer, SPIE 3244 (1996).

D. Eimerl, J. M. Auerbach, and P. W. Milonni, J. Mod. Opt. 42, 1037 (1995).

M. D. Feit and A. M. Rubenchik, SPIE 5273, 74 (2004).

J. B. Trenholme, A. M. Rubenchik, and M. D. Feit, SPIE In Press (2005).

M. D. Feit, A. M. Rubenchik, and J. B. Trenholme, In press (2005).

B. C. Stuart, M. D. Feit, S. Herman, A. M. Rubenchik, B. W. Shore, and M. D. Perry, Physical Review B (Condensed Matter) 53, 1749 (1996).

18 M. D. Feit and A. M. Rubenchik, SPIE 5273, 74 (2003). 